\title{
Damage and failure evaluation of diamond wire for multi-wire sawing of hard stone blocks through modelling and numerical simulation
}

\author{
Daniel Gomes ${ }^{1 *}$, Andreia Araujo ${ }^{1}$, Rui Marques ${ }^{1}$, José Patricio ${ }^{2}$, Vitor Lopez ${ }^{2}$ and Raquel \\ M. Santos ${ }^{1,3}$ \\ ${ }^{1}$ Institute of Science and Innovation in Mechanical and Industrial Engineering (INEGI), Materials and \\ Composite Structures unit, Porto, Portugal \\ ${ }^{2}$ Diamond Service Portuguesa, Lda, Zona Industrial Lt.2 Apt. 61 Ec, Vila Viçosa, Portugal \\ ${ }^{3}$ Associated Laboratory of Energy, Transports and Aeronautics (LAETA), Faculty of Engineering, \\ Porto University, Porto, Portugal
}

\begin{abstract}
Diamond wires are high-speed, efficient and cost-effective stone cutting tools used both in quarries, to obtain large stone blocks, and in blockprocessing plants, to shape ornamental stones. Diamond wires are generally composed by a wire rope with evenly spaced diamond beads fixed by a polymer or rubber coating. A numerical model of the diamond wire was developed in Abaqus ${ }^{\mathrm{TM}}$ software aiming to study the damage and failure of the steel wire during the cutting process. The model is intended to support the development of this component with enhanced durability and damage resistance.

Previously in this work, a detailed three-dimensional (3D) and numerical model of a $7 \times 7$ wire rope was created, followed by experimental validation. The diamond wire model was then based on the wire rope model, with the addition of the polymer coating and the diamond beads. The developed diamond wire model presents an error smaller than $6 \%$ relative to the experimental tensile test, corresponding to a valid representation of the component. This model has practical significance for the mechanical evaluation of the diamond wire, supporting further developments, with special focus on its design and manufacturing, to achieve longer service life.
\end{abstract}

\section{Introduction}

Diamond wires are commonly used as a cutting tool for the extraction of large stone blocks at quarries and/or to shape ornamental stones in block processing plants. It is the most efficient tool for stone cutting, and it already has a long history in the processing of limestone. Recent scientific developments and technological advances in the manufacturing of tailored diamond beads allowed the use of higher cutting speeds and increased the durability of the traditional beads, decreasing the overall cutting costs. Diamond beads also allowed the application of wires in harder stones, as granite. However, these developments impose a higher load requirement on the diamond wire, leading to premature failures [1].

\footnotetext{
*Corresponding author: dgomes@,inegi.up.pt
} 
Diamond wires consist essentially of a high-strength wire rope, with diamond beads threaded on it. The beads are responsible for cutting the stone, and are accurately separated and fixed by a rubber or polymer coating. The diamond wire contains between 30 and 40 beads per meter, and it is pre-twisted during its production, which induces an axial rotation during the cutting operation.

An overview of the literature revealed few analytical and experimental studies based on the cutting of stone using diamond wires, as well as on the numerical simulation of the wire rope.

S. Turchetta et al. [2] published an analytical model of the forces acting in the diamond bed, which is dependent on the cutting force, pre-tension, and torsion of the diamond wire. In addition, B. C. Liu et al. [3] presented a calculation of the diamond wire sawing trajectory. Moreover, an experimental study on the breakage of the diamond wire during the cutting operation was performed by $\mathrm{G}$. Huang and X. Xu [4]. The authors reported that the breakages were mainly induced by fatigue failure and occur mainly in two sections of the diamond wire, at the bead end and midsection between two beads.

G. A. Costello [5] developed an analytical model that enables the calculation of the stresses and deformation of each individual wire in response to the applied force and torsion. A mathematical model to define the complex geometry of wire rope is presented by X.-Y. Wang et al. [6], and numerical models with finite element simulation of the axial loading of an independent wire rope core (IWRC) are presented by C. Erdonmez and C. Ímrak [7]. The presented numerical model achieved a good correlation with the experimental results.

The focus of this work is the diamond wires used in multi-wire machines. These machines can have up to 90 diamond wires of $20 \mathrm{~m}$ each, which cut a large stone block into slabs of various thicknesses. Multi-wire machines have various configurations, but the main structure is composed of two large pulleys with a dimension of approximately $2 \mathrm{~m}$ in diameter. Two smaller guide pulleys are positioned near the rock with the function to align the diamond wire for the cutting operation. The motion of the wire is imposed by one of the large pulleys, the driving pulley, while the other large pulley works as a tensioner. In different machine models, the tension on the wire is imposed by a separate set of pulleys, positioned in the top section of the wire path. The rock block is positioned under this structure and, during the cutting operation, the whole structure moves downwards, in the direction of cutting the stone block, while the wire rotates tangential to the rock. In a multi-wire stone cutting machine from Hedel, the diamond wire runs at $30 \mathrm{~m} / \mathrm{s}$, with a pre-tension of around $180 \mathrm{kgf}$, with a down feed speed of $25 \mathrm{~cm} / \mathrm{h}$, taking around $8 \mathrm{~h}$ for a single block that normally ranges in the $3 \mathrm{~m}$ long and $1.5 \mathrm{~m}$ high.

Up to our knowledge, there is no literature regarding the numerical simulation of a diamond wire. This work aims to develop a numerical model of the diamond wire that correctly represents its stress/strain response during the cutting operation, applying the wire rope numerical simulation methods and the knowledge from the forces applied to the diamond wire and its behaviour on a multi-wire sawing machine.

\section{3D Modeling of the diamond wire}

\subsection{Wire rope}

The wire rope that composes the diamond wire has a $7 \times 7$ right regular lay. This $7 x 7$ construction consists of a central strand having another 6 laid around it, and each of those strands also has a central wire with 6 wires laid around. The right regular lay means that the outer strands and the wires from the central strand are winded clockwise while the wires from 
the outer strands are winded in the opposite direction. The diameters and pitch lengths of each wire and strand are presented in Fig. 1.

\begin{tabular}{|ll|}
\hline \multicolumn{2}{|c|}{ Radius (mm) } \\
\hline Wire rope & 1.575 \\
Strand 1 & 0.56 \\
Strand 2 & 0.52 \\
R1 & 0.22 \\
R2 & 0.17 \\
R3 & 0.19 \\
R4 & 0.165 \\
\hline \multicolumn{2}{|c|}{ Pitch (mm) } \\
\hline Outer Strand & 21.5 \\
Strand 1 wires & 7 \\
Strand 2 wires & 7 \\
\hline
\end{tabular}

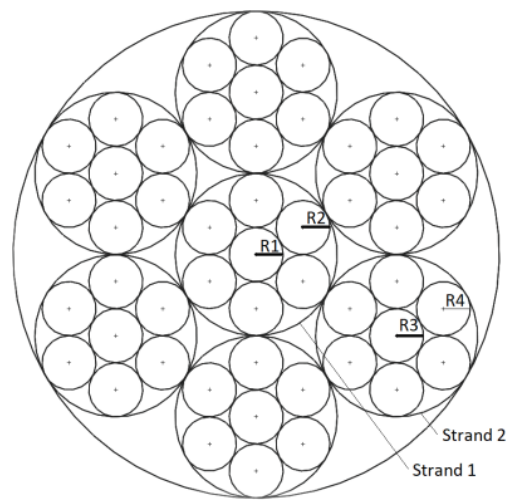

Fig. 1. Wire rope geometric dimensions.

A generic 3D modelling software, such as Soliworks ${ }^{\mathrm{TM}}$, was used to create the 3D model of the wire rope and the whole diamond wire. The single helical wires of the wire rope (outer wires of the central strand and central wire of the outer strands) were obtained through the extrude sweep of a circular profile with a helix as the path of extrusion that defined the pitch of the wire. While for the double helix wires (outer wires of the outer strands), a sketch of the 6 wires needed to be created. Afterwards, this sketch was extruded along the outer helix and a twist value was defined, to obtain the double helix wires. The wire rope was defined by 9 different individual wires, that can be patterned around the axis of the wire rope to create all 49 wires.

In the literature, the numerical models of wire rope are defined to have a rope length between 3 to $9 \%$ of the outer strands pitch [7]. However, the diamond wire model should have a representative length in accordance with the bead's length, and at the same time, it should be as short as possible, to be computationally feasible. Therefore, the diamond wire model was defined to represent a single diamond bead, and a length equal to the pitch between consecutive beads, which means that the wire rope model should have a $27 \mathrm{~mm}$ length (which is more than 1 pitch length of the wire rope).

The larger length of the wire rope, compared to previous numerical models, represents an additional challenge considering the non-uniform cross-section and consequent non-uniform diameter of a single strand, and its own twisting around the centre strand. If 2 wires with circular cross-sections are wounded around each other in helices, as for the single strands, the contact between both wires is a continuous line. On the other hand, in the steel cable, when the outer strands are wounded around the centre strand in regular lay construction, there is only contact when the outer wires of each strand cross each other (wires R2 and R4, Fig. 1). Since the 3D model uses helix paths, it only presents contact points in between the strands exactly at these locations, creating large gaps along the length of the wire rope. Observing both the 3D model and the deformation under a tensile stress of the wire rope model, it was considered that in the production of the steel cable some of these gaps are eliminated, due to the tension in the process and the tightened of the outer strands against the core strand. This assumption means that the larger gaps are not formed, creating new contact points.

The existence of the non-eliminated gaps allows the extension of the outer strands in tensile loading until contact between the two strands is achieved. This free body motion of the strands leads to a decrease in the stiffness of the wire rope model compared to the experimental results, as it can be observed in Fig. 2. To overcome this problem in the definition of the numerical model, a tie constraint was used between the outer wires of the 
core strand, and the outer wires of the outer strands. This constraint is only applied to the nodes of these surfaces that are within a specified distance of each other that is defined by the gaps dimension, to ensure that the outer strands behave as if any gaps to the centre strand are presented, and are not over-constrained.

The wire rope model was validated in relation to the analytical model of A. Costello [5]. As referred previously, in Fig. 2, a lack of stiffness of the wire rope model without tie constraint can be observed, which does not occur to the model with the specified distance tie. The final wire rope model is in good agreement with the analytical model of A. Costello [5], until the plastic deformation is achieved, since the analytical model does not account the plastic plateau of the material.

The material's properties used in the model included: young's modulus, $E=180000$ $\mathrm{MPa}$; yield stress, $\mathrm{Rp}_{0.2}=2160 \mathrm{MPa}$; and the plastic deformation was considered as perfectly plastic.

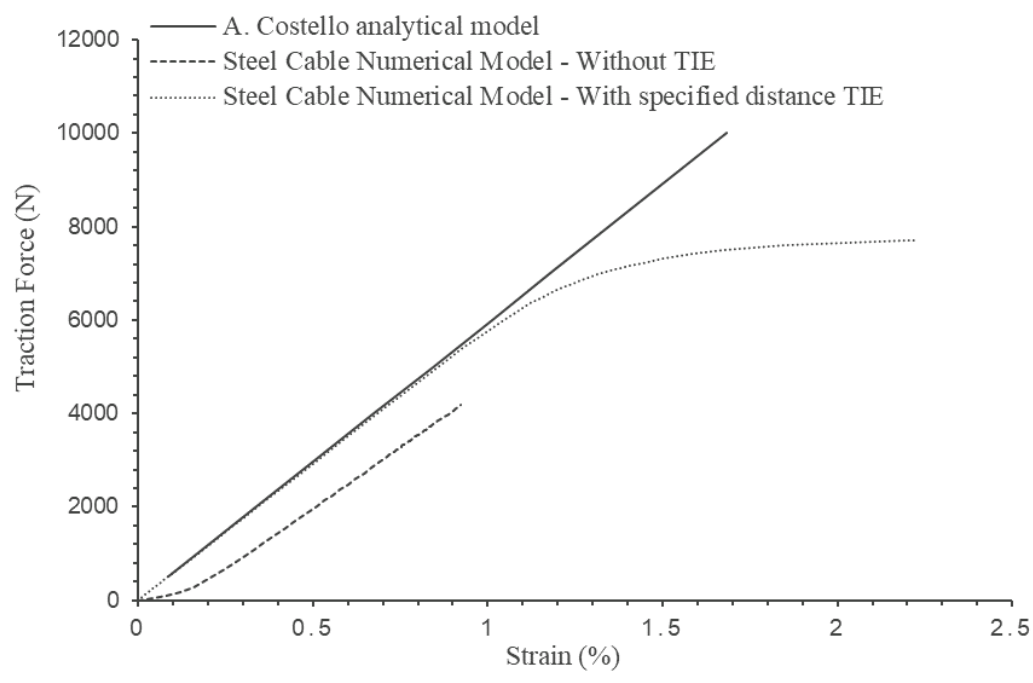

Fig. 2. Wire rope numerical model validation.

\subsection{Coating material}

The coating material used in this work was a thermoplastic polyurethane (TPU), which is an elastomer characterized by a hyperelastic behaviour [8]. The tensile and compression experiments were conducted to obtain the characteristic loading curve of the material, and the experimental results were imported to Abaqus ${ }^{\mathrm{TM}}$. In a first step, the evaluate function was used to select the strain energy potential that correctly modelled the material's behaviour. Afterwards, the different models of the material were used to replicate the experimental tensile and compression results in Abaqus ${ }^{\mathrm{TM}}$. According to the developed work, the Van der Waals model presented the best relation between the correct modelling of the material behaviour and computational efficiency. These findings are not presented in this work considering industrial confidentiality agreements.

\subsection{Diamond wire model}

For the diamond wire 3D model, a hollow circular profile, having $4.4 \mathrm{~mm}$ of outside diameter and $2.16 \mathrm{~mm}$ inside, was extruded along the axis of the wire rope to create the polymer 
coating. Then, the same function of sweep, although as cut-extrude, was used to create the wire rope negative geometry on the inside of the polymer coating. The diamond bead was inserted into the part and the function combine provided the negative geometry of the diamond bed on the outside of the polymer coating. The assembly of the wire rope, polymer coating, and diamond bead are presented in Fig. 3.
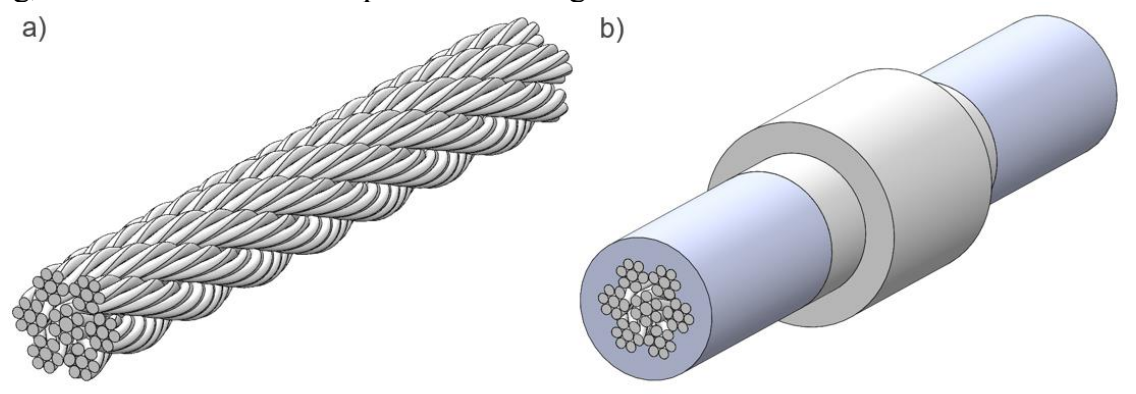

Fig. 3. a) Wire rope 3D model; b) Diamond wire 3D model assembly.

To validate the numerical model, tensile tests were carried out using diamond wires and a corresponding numerical simulation was used to compare the results. A good correlation between both the numerical model and the experimental test results was attained, with an error smaller than $6 \%$.

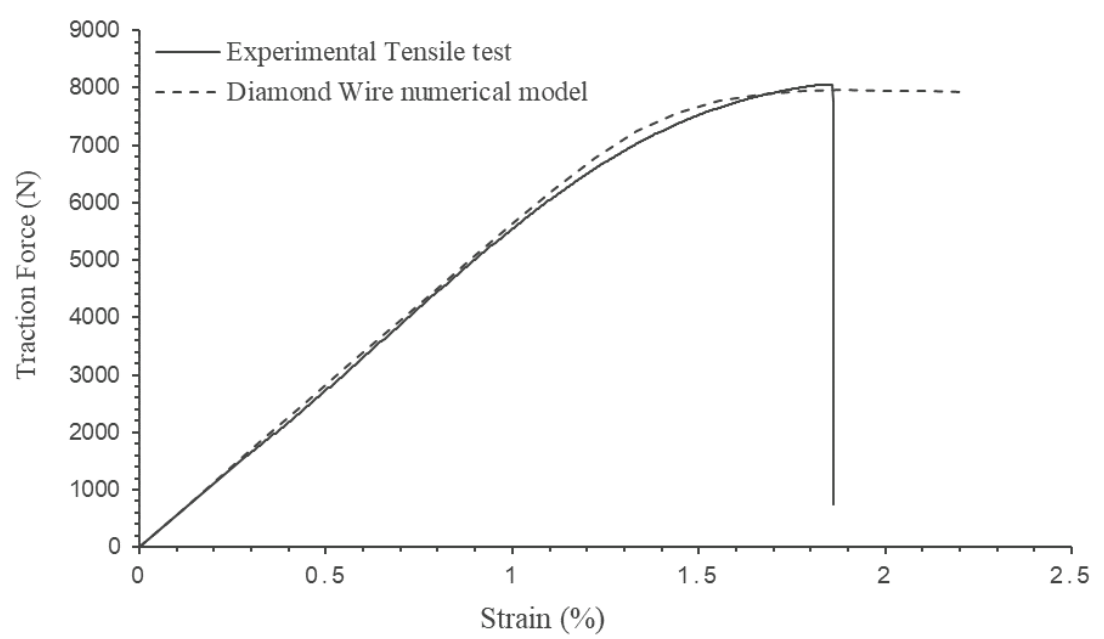

Fig. 4. Diamond wire numerical model validation.

\subsection{Stone cutting load case}

In a multi-wire stone cutting machine, the wire is subjected to different mechanical solicitations, including i) a pre-tension applied on the assembly of the cable by a tensioning pulley of the multi-wire sawing machine, ii) a tension applied by the driving pulley related to the cutting force, iii) a pre-torsion applied during cable production, and iv) bending when passing over a pulley or the stone surface. The tension has a maximum value of $2000 \mathrm{~N}$, the applied torsion is 1.5 turns per meter of diamond wire, corresponding to an angular deformation of $9.42 \mathrm{rad} / \mathrm{m}$, and the bending is dependent on the passing pulley. For a Hedel machine, the smallest pulley has a diameter of $780 \mathrm{~mm}$, corresponding to the highest bending stress. 
To replicate these loading conditions in the simulation, the diamond bead is constrained by a generating line of the bead. Two more points of the bead are restricted on the plane orthogonal to the axis to avoid its rotation around the fixed generating line. With the fixed bead, all stresses are applied to a reference point at the edges of the diamond wire model by applying: i) a $2000 \mathrm{~N}$ point load in the traction direction, ii) an angular displacement around the axis of 0.1272 radians, and iii) a displacement of $0.23 \mathrm{~mm}$ orthogonally applied to the axis of the wire and in a plane containing the fixed generating line, as well as the axis of the bead that represents the bending stress.

To evaluate the influence of each stress, they were divided into 3 consecutive steps: i) the first step only applied the point load, ii) the second step introduced the angular displacement, and iii) in the third step, the displacement imposing the bending stress was added.

\section{Results and Discussion}

The finite element simulation of the diamond wire, subjected to the presented stone cutting load case, is displayed in Fig. 5.
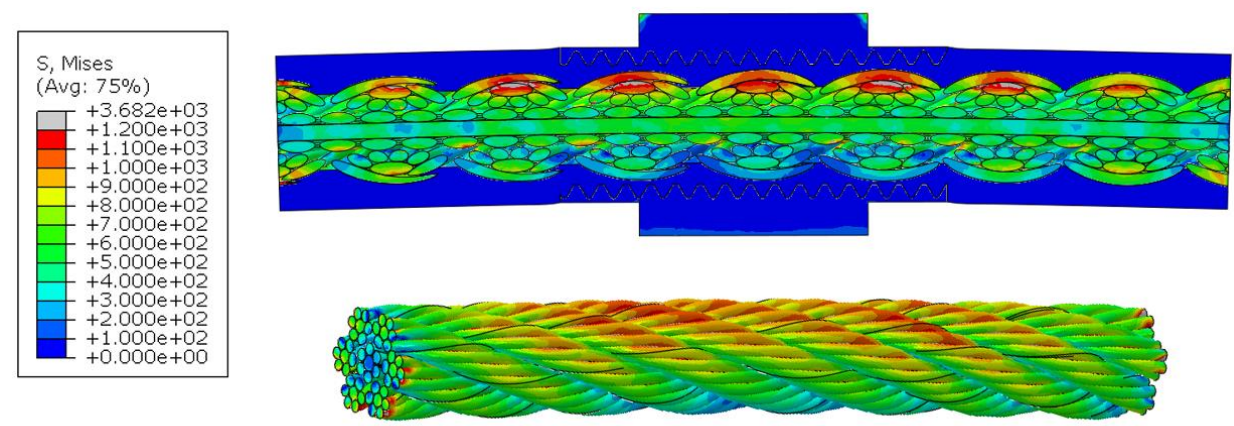

Fig. 5. Stress distribution on the wire rope subjected to the cutting load case.

The superposition of the tension and bending stress on the top wires of the wire rope creates a von mises equivalent stress of $1200 \mathrm{MPa}$, while in the compression side of the bending stress, the same wires present stresses averaging $500 \mathrm{MPa}$. The remaining wires of the wire rope show stress values between 600 and $700 \mathrm{MPa}$. With a different stress scale, as displayed in Fig. 6, the stress distribution on the TPU coating can be evaluated. This component, as defined in the bibliography, presents a stress concentration on the extremes of the bead [4], mainly on the first fillet of the bead thread. The reduction of these local stresses is one of the points in development to improve the service life of the diamond wire.

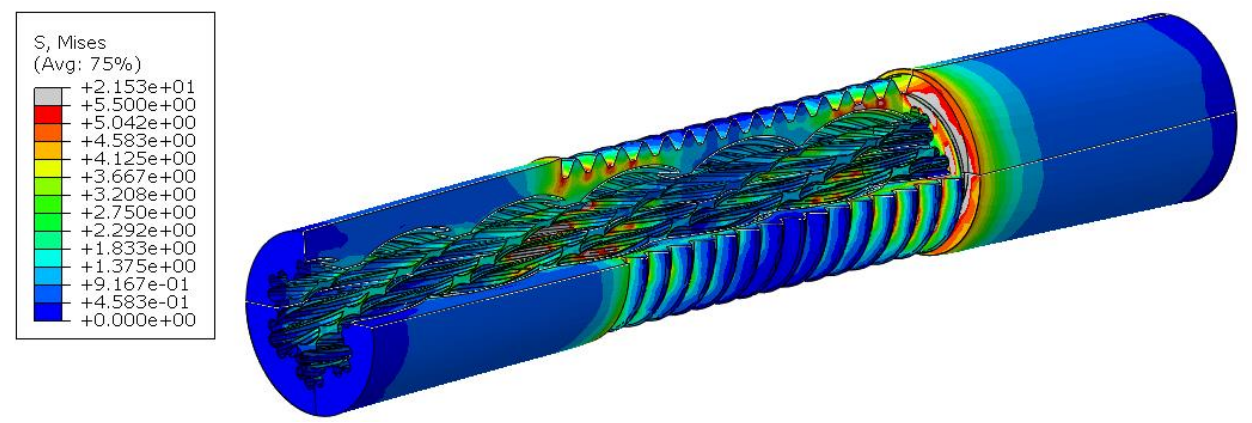

Fig. 6. Stress distribution on the TPU coating subjected to the cutting load case. 
Different loading cases were applied to the diamond wire model to analyse the influence of the cutting parameters. It was found that the pre-torsion applied to the cable during manufacturing has a great influence on the load distribution between the core and the outer strands of the steel wire. With the standard pre-torsion of 1.5 turns per meter, the torsion torque presented by the diamond wire model is $861 \mathrm{Nmm}$. The reduction of the pre-torsion value by $33 \%$ reduces the torsion torque in $16 \%$, but achieves a more homogenous stress distribution between the core and the outer strands as it can be seen in Fig. 7.

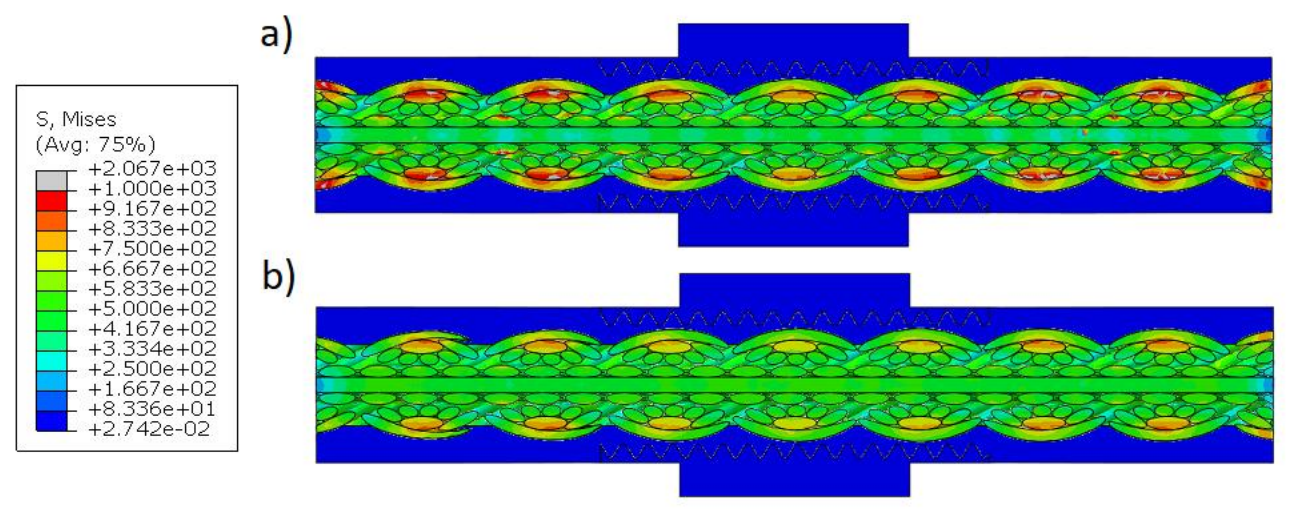

Fig. 7 Stress distribution on the diamond wire steel cable with a pre-torsion of a) 1.5 turns per meter and b) 1 turn per meter.

The pre-torsion is applied to the diamond wire during the production process to impose a torsion torque on the diamond wire, forcing the beads to rotate during the cutting operation, allowing the constant wear of the beads around their circumference. Moreover, the torsion torque applied in the diamond wire should be enough to impose the beads rotation. Although this can only be verified in experimental tests, the model allows the prediction of torsion torque loss relatively to the applied forces and the production parameters.

\section{Conclusions}

In this work, a diamond wire numerical model for finite element simulation was created with the use of the Abaqus ${ }^{\mathrm{TM}}$ software, allowing the correct evaluation of the stresses presented on the diamond wire during the cutting operation. It is possible to observe the stress's amplitude and distribution for each individual wire of the wire rope, for the TPU coating and for the diamond bead, in correlation with the different loading conditions applied to the diamond wire.

The model is extremely useful to understand the damage mechanisms present in the diamond wire, and the most relevant parameters that contribute for mechanical failure. For the further development of the diamond wire, it is possible to vary geometrical and material characteristics on the model and evaluate its influence on the stress's distribution. The appropriate solutions can then be implemented in the diamond wire to improve its service life. 


\section{References}

1. O. Cai, N. Careddu, M. Mereu, and I. Mulas, "The influence of operating parameters on the total productivity of diamond wire in cutting granite", Industrial Diamond Review, vol. 67, (2007).

2. S. Turchetta, L. Sorrentino, and C. Bellini, "A method to optimize the diamond wire cutting process," Diamond and Related Materials, vol. 71, pp. 90-97, (2017).

3. B. C. Liu, Z. P. Zhang, and Y. H. Sun, "Sawing Trajectory and Mechanism of Diamond Wire Saw," in Key Engineering Materials, vol. 258-259, pp. 395-400, (2004).

4. G. Huang and X. Xu, "Analysis of the Breakage of Diamond Wire Saws in Sawing of Stone," in Key Engineering Materials, vol. 304-305, pp. 123-126, (2006).

5. G. A. Costello, Theory of wire rope, Springer Science \& Business Media, (1997).

6. X.-Y. Wang, X.-B. Meng, J.-X. Wang, Y.-H. Sun, and K. Gao, "Mathematical modeling and geometric analysis for wire rope strands," Applied Mathematical Modelling, vol. 39, no. 3, pp. 1019-1032, (2015).

7. C. Erdonmez and C. E. Imrak, "A finite element model for independent wire rope core with double helical geometry subjected to axial loads," Sadhana, vol. 36, no. 6, pp. 995-1008, (2011).

8. H. J. Qi and M. C. Boyce, "Stress-strain behavior of thermoplastic polyurethane," Mechanics of Materials, 37(8): p. 817-839, (2005). 